\title{
Requirements and standards facilitating quality improvement for reporting systems in gastrointestinal endoscopy: European Society of Gastrointestinal Endoscopy (ESGE) Position Statement

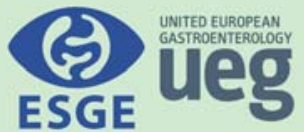

Authors

Institutions
Michael Bretthauer ${ }^{1,2}$, Lars Aabakken ${ }^{2}$, Evelien Dekker ${ }^{3}$, Michal F. Kaminski ${ }^{1,4}$, Thomas Rösch ${ }^{5}$, Rolf Hultcrantz ${ }^{6}$, Stepan Suchanek ${ }^{7}$, Rodrigo Jover ${ }^{8}$, Ernst J. Kuipers ${ }^{9}$, Raf Bisschops ${ }^{10}$, Cristiano Spada ${ }^{11}$, Roland Valori ${ }^{12}$, Dirk Domagk ${ }^{13}$, Colin Rees $^{14,15}$, Matthew D. Rutter ${ }^{14,15}$, on behalf of the ESGE Quality Improvement Committee

Institutions are listed at end of article.
Bibliography

DOI http://dx.doi.org/

$10.1055 / \mathrm{s}-0042-100186$

Published online: 3.2.2016

Endoscopy 2016; 48: 291-294

(c) Georg Thieme Verlag KG

Stuttgart · New York

ISSN 0013-726X

\section{Corresponding author}

Michael Bretthauer, MD, PhD

University of Oslo

Oslo

Norway

michael.bretthauer@medisin. uio.no

This article is published simultaneously in the journals Endoscopy and the United European Gastroenterology Journal. Copyright 2016 (c) Georg Thieme Verlag KG and $\odot$ by the United European Gastroenterology

To develop standards for high quality in gastrointestinal (GI) endoscopy, the European Society of Gastrointestinal Endoscopy (ESGE) has established the ESGE Quality Improvement Committee. A prerequisite for quality assurance and improvement for all GI endoscopy procedures is state-of-the-art integrated digital reporting systems for standardized documentation of the procedures. The current paper describes the ESGE's viewpoints on the requirements for high-quality endoscopy reporting systems in GI endoscopy.

\section{Recommendations}

1 Endoscopy reporting systems must be electronic. 2 Endoscopy reporting systems should be integrated into hospitals' patient record systems.

3 Endoscopy reporting systems should include patient identifiers to facilitate data linkage to other data sources.

4 Endoscopy reporting systems shall restrict the use of free-text entry to a minimum, and be based mainly on structured data entry.

5 Separate entry of data for quality or research purposes is discouraged. Automatic data transfer for quality and research purposes must be facilitated. 6 Double entry of data by the endoscopist or associate personnel is discouraged. Available data from outside sources (administrative or medical) must be made available automatically.

\section{Abbreviations}

$\nabla$

ESGE European Society of Gastrointestinal Endoscopy

ICD International Classification of Diseases

GI gastrointestinal

MST Minimal Standard Terminology

SNOMED CT Systematized Nomenclature of Medicine - Clinical Terms

WHO World Health Organization
7 Endoscopy reporting systems shall facilitate the inclusion of information on histopathology of detected lesions, patient satisfaction, adverse events, and surveillance recommendations.

8 Endoscopy reporting systems must facilitate easy data retrieval at any time in a universally compatible format.

9 Endoscopy reporting systems must include data fields for key performance indicators as defined by quality improvement committees.

10 Endoscopy reporting systems must facilitate changes in indicators and data entry fields as required by professional organizations.

\section{Introduction \\ $\nabla$}

The endoscopy report is central to any endoscopy practice and facilitates the exchange of information about findings, therapy, clinical recommendations, adverse events, and performance in relation to endoscopy procedures.

Similar to medical records in general, endoscopy reports have traditionally used free-text or unstructured-text phrases, occasionally accompanied by photo-documentation. Free-text phrases, however, prevent meaningful data extraction and are therefore a barrier to quality assurance in GI endoscopy.

In recent years much effort has been put into the creation of a comprehensive terminology and coding system for GI endoscopy [1]. The system, 
which has been approved by the WHO, is based on the 10th edition of the International Classification of Diseases (ICD-10) and allows the description of every possible GI endoscopic term. This has created a platform to standardize language and terms used in GI endoscopy. As a result, minimal standard terminology (MST) and other initiatives, such as SNOMED CT (Systematized Nomenclature of Medicine - Clinical Terms; see http://www. ihtsdo.org/. Accessed: 11 December 2015), have evolved to aid the unification of endoscopy reporting within and between countries [2-4].

Adequate monitoring of GI endoscopy and the assurance of high quality in endoscopy services require that data extraction is easily performed from endoscopy reports of procedural data, patient characteristics, and key quality indicators [5]. Implementation of quality-assured and standardized endoscopy reporting systems in daily practice is feasible [5].

The European Society of Gastrointestinal Endoscopy (ESGE) has established a quality improvement committee to facilitate improvement in the global quality of endoscopy and the delivery of patient-centered endoscopy services, to promote within ESGE activities a unifying theme of quality in endoscopy, and to assist endoscopy units and endoscopists in achieving these standards. Among other activities, the committee will develop quality standards, guidance, and position statements to promote quality in endoscopy.

The present paper describes the views of the ESGE on the requirements and standards for endoscopy reporting systems. The recommendations are summarized in $\bullet$ Table 1.

\section{Methods}

$\nabla$

The members of the ESGE Quality Improvement Committee were appointed by the ESGE governing board after recommendation by the ESGE national societies. The authors of this paper are the chairs of all the subcommittees of the ESGE Quality Improvement Committee and the members of the task force "Reporting systems in gastrointestinal endoscopy" under the subcommittee "Lower GI endoscopy."

A literature search on the topics described in this article was performed in February 2015 (by M.B.). Identified literature was reviewed and discussed among the authors.

Image documentation by still photographs and videos are an important part of endoscopy reporting systems, but are not a topic of the present article; this paper focuses on the written features of endoscopy reports. The present article is a "position statement" because there is a lack of adequate literature to support grading of the recommendations. The views expressed in this paper are derived by consensus among the authors. The final draft of the paper was sent for review to two ESGE governing board members for review.

\section{Electronic or paper-based reporting}

$\nabla$

\section{Endoscopy reporting systems must be electronic.}

Modern endoscopy reporting systems should be digital to permit continuous data monitoring. Electronic reporting, and storage of text and images allows continuous monitoring for quality purposes at endoscopist, unit, regional, and national level. Further-
Table 1 Recommendations: requirements for future endoscopic reporting systems.

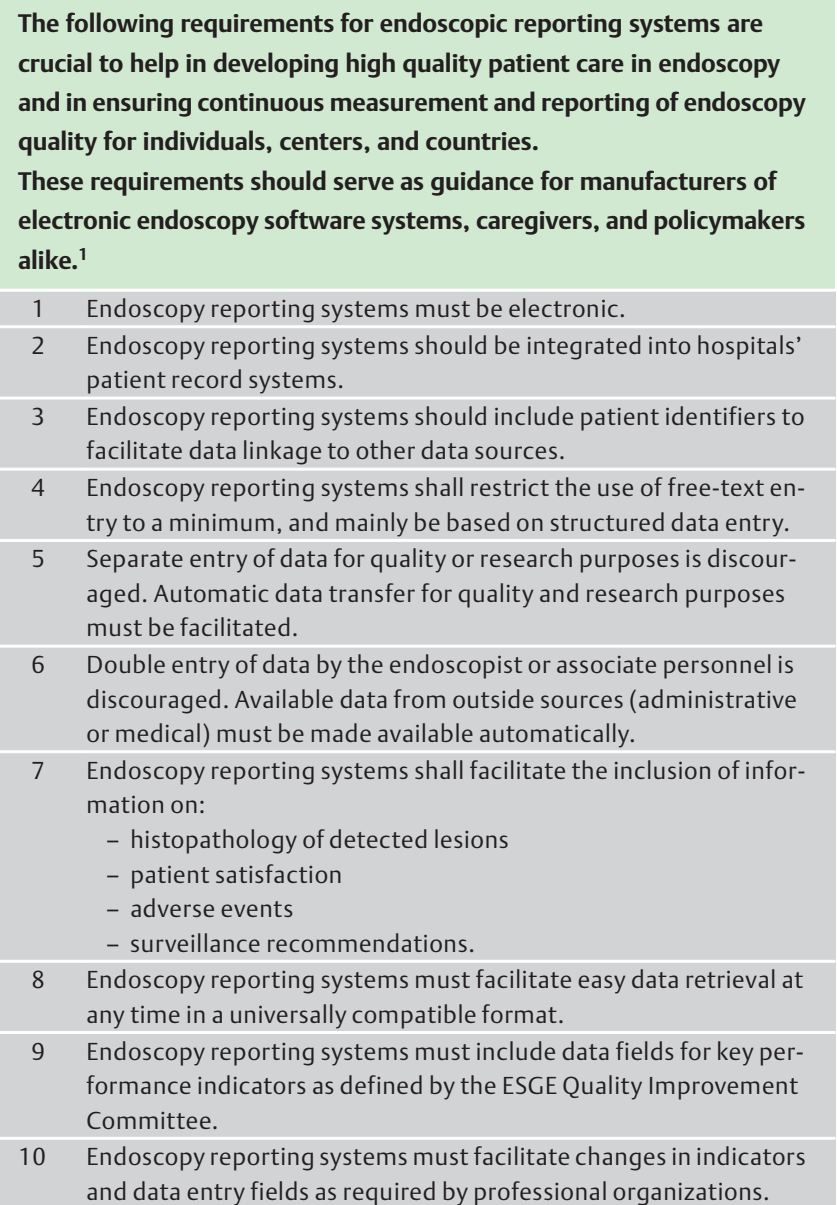

${ }^{1}$ Image documentation by still photographs and videos are an important part of endoscopy reporting systems, but are not a topic of the present article; these recommendations focus on the written features of endoscopy reports.

more, electronic reporting facilitates continuous access for all involved in the clinical management of individual patients, e.g. for multidisciplinary teams, and for audit of complications and adverse events. It also enables the comparison of digital images from repeated procedures.

Finally, comprehensive endoscopy reporting systems, when coupled with disinfection machines, allow adequate tracking and tracing of equipment for the early detection of potential flaws in disinfection and reprocessing.

For all of these reasons, digital reporting has been shown to be cost-efficient [6] and is a prerequisite for universal quality reporting.

\section{Integrated or stand-alone reporting}

Integ

Endoscopy reporting systems should be integrated into hospitals' patient record systems.

Endoscopy reporting systems should include patient identifiers to facilitate data linkage to other data sources. 
Many endoscopy practices, either in hospitals or stand-alone, have institutional electronic patient record systems. Endoscopy reporting systems should ideally be integrated into these systems to facilitate their usefulness to other medical professionals and to allow data exchange between the endoscopy and patient record systems within the hospital and between connected hospitals.

Often, however, the manufacturers of institutional patient records systems do not have an endoscopy reporting module in their portfolio. Providers of endoscopy reporting systems should ensure that their system communicates seamlessly with the main patient record systems of the hospital or institution. The main priority is to improve the quality of endoscopy reporting without affecting the collection of the administrative and patient data needed to monitor the performance and quality of the endoscopy services.

\section{Structured and standardized data entry} $\boldsymbol{\nabla}$

Endoscopy reporting systems shall restrict the use of free-text entry to a minimum, and be based mainly on structured data entry.

Free-text reporting is strongly discouraged because it leads to incomplete data and low quality reporting of endoscopies [7-10]. Endoscopy reporting systems need to use structured terminology whenever possible (in concordance with validated, standardized terminology), and limit the use of free-text data entry. Free text should be restricted to individualized clinical recommendations at the end of the endoscopy report, addressing all relevant clinical questions. Other free-text fields are usually not required. The software of the endoscopy reporting systems needs therefore to produce readable endoscopy reports for readers who are not specialists in endoscopy. A good report takes time to put together. Thus, clinics need to provide time to allow for the training of personnel to learn the application of electronic reporting systems.

\section{Variables}

$\boldsymbol{\nabla}$

Separate entry of data for quality or research purposes is discouraged. Automatic data transfer for quality and research purposes must be facilitated.

Endoscopy reporting systems must include data fields for key performance indicators as defined by the ESGE Quality Improvement Committee.

A number of standards have been established for the required contents of endoscopy reports $[3,11]$. These have usually been developed by clinical endoscopy interest groups, quality improvement groups, or researchers. It is our viewpoint that there is no inherent conflict between these three and endoscopy reporting systems need to facilitate a minimum number of variables derived from all these three domains. Indeed, many of the required variables will relate to more than one of the domains. Rather than defining variables as clinical, quality, or research, a minimal standard variable list should be defined for each procedure and should be included in endoscopy reporting systems.

Endoscopy reporting systems also need to be able to allow extension of variables, as desired by local users on a case-by-case basis and over time as the specialty of endoscopy advances. The minimal variable list should be uniform across all systems to facilitate data exchange and the monitoring of clinical services, quality improvement, and research across endoscopists, units, and countries. We strongly encourage manufacturers to adhere to these standards.

Within the next 2 years, the ESGE Quality Improvement Committee will provide minimal variable lists for each type of endoscopy procedure within the framework of the subcommittees for upper GI endoscopy, lower GI endoscopy, pancreatobiliary, and smallbowel endoscopy, respectively.

\section{Data entry \\ $\nabla$}

Double entry of data by the endoscopist or associate personnel is discouraged. Available data from outside sources (administrative or medical) must be made available automatically.

Endoscopy reporting systems shall facilitate the inclusion of information on histopathology of detected lesions, patient satisfaction, adverse events, and surveillance recommendations.

Double entry of data, as currently occurs for quality improvement or research, is the principal barrier to obtaining quality-assured reporting in daily practice. It therefore impedes continuous quality improvement and the acquisition of important research data, and can be a potential source of mistakes. Future endoscopy reporting systems need to be structured in such a way as to enable reliable data entry and easy extraction of performance reports for quality improvement and research. Double data entry should not be needed in the current era and is strongly discouraged.

Data such as histopathology results, patient comfort, patient satisfaction, and post-procedure adverse events should become an integral part of future endoscopy reporting systems. This can be achieved by automatic linkage between the endoscopy reporting system and other databases using unique patient identifiers.

Endoscopy systems should have an integrated quality assurance module. For example, a tracking system is necessary to monitor histopathology results to ensure that each resected lesion is clearly described (size, location, completeness of resection, container number), entered into the system, and signed off by the responsible endoscopist. Quality control can be achieved by regular automated cross-checks with warnings generated when performance falls outside accepted parameters. Terminology and quality standards from other specialties, such as histopathology, need to be adapted through consensus between the different specialties.

\section{Data output}

$\boldsymbol{\nabla}$

Endoscopy reporting systems must facilitate easy data retrieval at any time in a universally compatible format.

Endoscopy reporting systems must have the functionality to allow automated data extraction to provide predefined reports of clinical performance, quality indicators (which will be provided by the ESGE committees), and research data. Ideally, systems 
should allow local teams to develop their own customized data output reports.

Patient and procedure characteristics such as patient age and sex, indication for the procedure, preparation, and previous surgery are important to interpret variations in performance between individual endoscopists. Therefore, endoscopy reporting systems need to be able to provide different thresholds of acceptable performance based on case mix and other confounding patient and procedure characteristics. For each patient, all variables registered need to be available for data analysis to monitor quality for research purposes. All data need to be easily transferable to spreadsheet and standard statistical software packages.

\section{Continuous updating \\ $\nabla$}

Endoscopy reporting systems must facilitate changes in indicators and data entry fields as required by professional organizations.

The development of more widespread and effective quality assurance along with new evidence are likely to generate new quality variables. Therefore endoscopy reporting systems should be structured to enable changes, particularly the incorporation of new variables, to be made without requiring major rewrites of the software.

\section{The quality of quality control}

$\nabla$

Comprehensive quality measurements require manual data collection or linkages between several data systems (depending on safety regulation barriers). For quality improvement to itself be of high quality requires that all these interacting databases be of high quality. A recent study based on administrative databases showed that of 45 reported interval colon cancers, 21 were found to be administrative errors after careful checks were made of the patient records [12].

The issue of quality control of quality control systems is a challenge that will be particularly important when quality drives reimbursement (pay for performance). Medical societies themselves have to establish rules on how to deal with these issues before they are installed by other bodies. The committee aims to contribute to this issue in its future work.

Competing interests: Michael Bretthauer receives funds from Thieme Verlag for editorial work for Endoscopy. Evelien Dekker's department receives research support and equipment loans from Olympus Europe (for the last 10 years). Raf Bisschops has received: speaker's fees from Covidien (2009-2014) and Fujifilm (2013); a speaker's fee and hands-on training sponsorship from Olympus Europe (2013 - 2014); a speaker's fee and research support from Pentax Europe; and an editorial fee from Thieme Verlag as co-editor of Endoscopy. Cristiano Spada has received training support from Given Imaging (2013 and 2014). Roland Valori is a director of Quality Solutions for Healthcare, a company providing consultancy for improving quality and training in healthcare. Colin Rees's department receives research funding from Olympus Medical, ARC Medical, Aquilant Endoscopy, Almirall, and Cook (from 2010 to the present). Matthew D. Rutter's department receives research funding from Olympus for a colitis surveillance trial (2014 to present). Lars Aabakken, Dirk Domagk, Rolf Hultcrantz, Rodrigo Jover, Michal F. Kaminski, Ernst J. Kuipers, Thomas Rösch and Stepan Suchanek have no competing interests.

\section{Institutions}

${ }^{1}$ Department of Health Management and Health Economy and KG Jebsen Centre for Colorectal Cancer, University of Oslo, Oslo, Norway

2 Department of Transplantation Medicine, Oslo University Hospital, Oslo, Norway

${ }^{3}$ Department of Gastroenterology and Hepatology, Academic Medical Center, University of Amsterdam, Amsterdam, The Netherlands

${ }^{4}$ Department of Gastroenterological Oncology, The Maria Sklodowska-Curie Memorial Cancer Centre and Institute of Oncology, and Medical Center for Postgraduate Education, Warsaw, Poland

5 Department of Interdisciplinary Endoscopy, University Hospital HamburgEppendorf, Germany

${ }^{6}$ Karolinska Institute and Karolinska University Hospital, Stockholm, Sweden Department of Internal Medicine, First Faculty of Medicine, Charles University, Military University Hospital Prague, Czech Republic

${ }^{8}$ Unidad de Gastroenterologia, Hospital General Universitario de Alicante, Alicante, Spain

${ }^{9}$ Department of Gastroenterology and Hepatology, Erasmus MC University Medical Center, Rotterdam, the Netherlands

${ }^{10}$ Gastroenterology Department, University Hospital Leuven, Leuven, Belgium

${ }^{11}$ Digestive Endoscopy Unit, Catholic University, Rome, Italy

12 Department of Gastroenterology, Gloucestershire Royal Hospital, Gloucester, UK

${ }^{13}$ Department of Internal Medicine, Joseph's Hospital, Warendorf, Germany

${ }^{14}$ Department of Gastroenterology, University Hospital of North Tees, Stockton-on-Tees, Cleveland, UK

${ }^{15}$ School of Medicine, Durham University, Durham, UK

\section{Acknowledgement}

$\nabla$

ESGE and UEG have identified the quality of endoscopy as a major priority. UEG co-funded the ESGE quality improvement initiative and provided additional project governance.

\section{References}

1 Groenen MJ, Hirs W, Becker H et al. Gastrointestinal endoscopic terminology coding (GET-C): a WHO-approved extension of the ICD-10. Dig Dis Sci 2007; 52: 1004-1008

2 Delvaux M, Crespi M, Armengol-Miro JR et al. Minimal standard terminology for digestive endoscopy: results of prospective testing and validation in the GASTER project. Endoscopy 2000; 32: 345-355

3 Aabakken L, Rembacken B, LeMoine $O$ et al. Minimal standard terminology for gastrointestinal endoscopy - MST 3.0. Endoscopy 2009; 41: $727-728$

4 Aabakken L, Barkun AN, Cotton PB et al. Standardized endoscopic reporting. J Gastroenterol Hepatol 2014; 29: 234-240

5 van Doorn SC, van Vliet J, Fockens $P$ et al. A novel colonoscopy reporting system enabling quality assurance. Endoscopy 2014; 46: 181-187

6 Groenen MJ, Ajodhia S, Wynstra JY et al. A cost-benefit analysis of endoscopy reporting methods: handwritten, dictated and computerized. Endoscopy 2009; 41: 603-609

7 Moorman PW, van Ginneken AM, van der Lei J et al. The contents of free-text endoscopy reports: an inventory and evaluation by peers. Endoscopy 1994; 26: $531-538$

8 de Lange T, Moum BA, Tholfsen JK et al. Standardization and quality of endoscopy text reports in ulcerative colitis. Endoscopy 2003; 35: 835 840

9 Aabakken L. Quality reporting - finally achievable? Endoscopy 2014; 46: $188-189$

10 Hoff G, Ottestad PM, Skafløtten SR et al. Quality assurance as an integrated part of the electronic medical record - a prototype applied for colonoscopy. Scand J Gastroenterol 2009; 44: 1259-1265

11 Lieberman D, Nadel M, Smith RA et al. Standardized colonoscopy reporting and data system: report of the Quality Assurance Task Group of the National Colorectal Cancer Roundtable. Gastrointest Endosc 2007; 65: 757-766

12 Gotfried J, Bernstein M, Ehrlich AC et al. Administrative database research overestimates the rate of interval colon cancer. J Clin Gastroenterol 2015; 49: $483-490$ 